\title{
Associations between psychosocial risk factors and musculoskeletal disorders: application to the IT profession in India
}

\author{
Ranjana K. Mehta ${ }^{\mathrm{a}^{*}}$ and Prakriti Parijat ${ }^{\mathrm{b}}$ \\ ${ }^{a}$ Departments of Cognitive and Learning Sciences and Kinesiology and Integrative Physiology, Michigan \\ Technological University, 1400 Townsend Drive, Houghton, MI, USA \\ ${ }^{\mathrm{b}}$ Human Factors International, India
}

\begin{abstract}
The exponential growth of the information technology (IT) industry in India has been accompanied with a substantial increase in the reporting of work-related musculoskeletal disorders (WMSDs). The purpose of the current study was to identify and assess the contributions of prevalent psychosocial factors on perceived WMSD outcomes among IT professionals from India. About 77 IT professionals from India completed a survey set consisting of 26 question items from the Job Content Questionnaire and 3 separate question items pertaining to WMSD outcomes (pain/discomfort and psychological stress scores). The findings suggest prevalence of existing pain (shoulder/neck and low back) in more than one-fourth of the respondents. Additionally two-thirds of the respondents had never had any ergonomics awareness training. Co-worker support and psychological work demands were found to be the strongest contributors of psychosocial risk factors towards pain/discomfort and psychological stress outcomes. Findings from this study highlight the influence of certain psychosocial traits of the Indian IT workplace on perceived WMSD outcomes. There is a need to develop and implement intervention strategies to address these factors that may help lower the risk of work-related musculoskeletal pain.
\end{abstract}

Keywords: psychosocial risk factors, musculoskeletal pain, information technology, social support

\section{Introduction}

The exponential growth of the information technology (IT) industry in India has been accompanied with a substantial increase in the reporting of workrelated musculoskeletal disorders (WMSDs). About $76 \%$ of computer professionals in India reported musculoskeletal pain and discomfort in various epidemiological studies $[3,24,26]$. In a recent study by Sharan et al. [23], $70 \%$ of computer professional reported pain and discomfort during and shortly after completing work with computer along with numbness/tingling sensation and loss of hand strength. These studies support the theory that WMSDs have a multifactorial etiology, with physical work demands, psychosocial risk factors, and individual differences contributing significantly to the cause of the disorder [31].

The pathophysiology of WMSDs due to physical risk factors, such as static work and awkward postures, has been investigated among Indian IT professionals [26]. However, the contributing effects of work-related psychosocial risk factors (that are prevalent in the IT industry in India) towards WMSD development remain unclear. Work-related psychosocial factors, often referred as work organizational factors, are defined as perceptions or belief of workers about the way their work environment is organized $[6,12,27,30]$. Psychosocial factors, that are associated with job and organizational environment (e.g., intensified workload, social support), have shown to influence biomechanical demands and result in adverse muscular and cardiovascular load

* Corresponding author. E-mail: ranjanam@mtu.edu 
$[17,21]$. However, only a few studies have reported associations between psychosocial factors prevalent in Indian work culture to musculoskeletal discomfort $[4,23]$. Furthermore, limited information is available that demonstrates direct association of WMSD outcomes due to psychosocial factors specifically among the Indian IT professionals.

Various questionnaires have been developed to evaluate and quantify psychosocial factors within specific occupations. For example, the Workstyle questionnaire quantifies worker's behavioral, physiological, and cognitive responses to a perceived high level of workload [9]. Several other questionnaires have been developed to assess workload, such as the NASA Task Load Index (NASA TLX) [11] and the Subjective Workload Assessment Technique (SWAT) questionnaires [22]. Specifically, the SWAT questionnaire consists of three dimensions, time load, mental effort, and psychological stress, which indicate the level of time pressure, mental workload, and stress associated with a given task. In order to evaluate musculoskeletal disorders, separate scales, such as the Borg CR10 scale, have been used to quantify pain/discomfort in localized joints or muscle sites [5]. Perceived pain or discomfort have been associated with increased prevalence of musculoskeletal disorders, and are thus often considered as perceived WMSD outcomes [7].

The Job Content Questionnaire (JCQ) focuses on quantifying the perception of psychosocial environment [14]. This questionnaire was developed from the need to identify work-related risk factors based on the job demand-control (job strain) model, developed by Karasek [13]. According to this model, high job demands, lower decision latitude, and low social support at work place workers at higher work stress (i.e., deteriorating psychological well-being) and increased risk of cardiovascular diseases. The JCQ consists of 49 items distributed across several scales: skill discretion, decision authority, psychological work demands, physical exertion, social support, and co-worker support. The JCQ scales has been previously employed to draw associations of psychosocial factors to cardiovascular diseases and WMSDs $[1,28]$, and have shown good internal reliability (Cronbach's $\alpha>0.70$ ) for most scales [15].

The JCQ has been internationally used to assess psychosocial job characteristics in different occupations [14]. However, only one study has employed the JCQ to assess psychosocial strains among Indian foundry shop floor workers [19]. The increasing focus on the development and expansion of the IT in- dustry in India initiates a need for the JCQ to be employed to assess psychosocial strain on this working sector. The purpose of the current study was to identify and assess the contributions of prevalent psychosocial factors on perceived WMSD outcomes among IT professionals from India. The study employed the Job Content Questionnaire to identify psychosocial risk factors prevalent in IT professionals in India, and to quantify associations between these psychosocial risk factors and WMSD outcomes.

\section{Methods}

The data presented in this study were extracted from various IT professional in Bangalore, India who responded to a survey sent via email. An online survey set was distributed among 200 IT professionals; 77 complete responses were obtained. The survey set contained questions from the JCQ, the SWAT questionnaire, and pain/discomfort scales.

\subsection{Measures}

Perceptions of the psychosocial environment were obtained used six scales of the JCQ, namely skill discretion (six items), decision authority (three items), psychological work demands (eight items), physical exertion (one item), supervisor support (four items), and co-worker support (four items). The JCQ has been successfully tested among different nationalities and between several occupations [14] and was thus believed to provide good internal reliability for the various scales in the target population. Scoring of these scales were conducted using instructions provided in the JCQ User's Guide [13]. Demographic data were also obtained from the respondents, which included age, gender, duration of computer use, amount and duration of breaks, and desktop/laptop user.

\subsection{WMSD outcomes}

The psychological stress scale from the SWAT questionnaire was employed to assess work stress (anchors ranging from $0=$ "little confusion, risk, frustration, or anxiety exists and can be easily accommodated", to 20 = "high to very intense stress due to confusion, frustration, or anxiety, with high to extreme determination and self-control required"), and pain/discomfort scales (measured using a 10-point scale ranging from $0=$ "no pain/discomfort" to $10=$ 
"extremely strong pain/discomfort") were employed to measure pain/discomfort in different body regions (hand/wrist, neck/shoulder, and upper/lower back) during and after work.

\subsection{Data analysis}

Prevalence of psychosocial risk factors, pain, and discomfort for the entire sample were determined using descriptive statistics. To analyze the relationship between demographic variables, psychosocial risk factors, and WMSD outcomes (i.e., pain and discomfort ratings), a non-parametric correlation analysis was performed between these items. Using step-wise regression, predictors of these WMSD outcomes were determined. Relevant non- parametric tests were employed wherever necessary. All statistical tests were considered significant when $\mathrm{p}<0.05$.

\section{Results}

\subsection{Demographic data}

The average age of participants was $27.1 \pm 4.3$ years. Of the respondents, $66 \%$ were males. The average daily work hours was $8.57 \pm 2.2$ hours; $42 \%$ respondents used laptops and $25 \%$ respondents used desktops for their daily work activities. A majority $(70 \%)$ of the respondents indicated that they took about 1-3 breaks daily, and 70\% indicated that the cumulative duration of breaks per day (excluding lunch break) was from 0-30 minutes. 43\% respondents reported minimal or no exercise, and only $5 \%$ respondents exercised almost daily. Of the 77 respondents, only $14 \%$ received any formal ergonomics training at their workplace. About $23-36 \%$ of the respondents used chairs that they felt were "ergonomically" comfortable or arm rest/supports that were at comfortable height. Furthermore, only about $7-10 \%$ respondents used additional wrist support for keyboard or mouse pad or any special ergonomic keyboards, such as the split keyboard.

About 29\% respondents indicated that they were suffering from previous health issues; specifically back pain $(n=14)$ and shoulder/neck pain $(n=5)$. Mean pain/discomfort and psychological stress scores indicated by respondents for various body parts "during" and "after" work are illustrated in Figure 1.
Table 1

Demographic data

\begin{tabular}{|c|c|c|}
\hline Variables & $\mathbf{N}$ & $\%$ \\
\hline \multicolumn{3}{|l|}{ Gender } \\
\hline Male & 51 & 66.2 \\
\hline Female & 26 & 33.1 \\
\hline \multicolumn{3}{|l|}{ Technology used } \\
\hline Laptop & 32 & 41.6 \\
\hline Desktop & 19 & 24.7 \\
\hline Both & 25 & 32.5 \\
\hline \multicolumn{3}{|l|}{ Duration of breaks } \\
\hline $0-30$ minutes & 56 & 72.7 \\
\hline $30-60$ minutes & 14 & 18.2 \\
\hline $1-2$ hours & 5 & 6.5 \\
\hline$>2$ hours & 1 & 1.3 \\
\hline \multicolumn{3}{|l|}{ Weekly exercise } \\
\hline Not at all & 33 & 42.9 \\
\hline Once a week & 10 & 13 \\
\hline Twice a week & 12 & 15.6 \\
\hline 3 - 4 times a week & 16 & 20.8 \\
\hline Almost everyday & 4 & 5.2 \\
\hline \multicolumn{3}{|c|}{ Existing health problem } \\
\hline Yes & 22 & 28.6 \\
\hline No & 46 & 59.7 \\
\hline Don't know & 6 & 7.8 \\
\hline \multicolumn{3}{|c|}{ Ergonomicstraining } \\
\hline Yes & 11 & 14.3 \\
\hline No & 59 & 76.6 \\
\hline Don't know & 3 & 3.9 \\
\hline
\end{tabular}

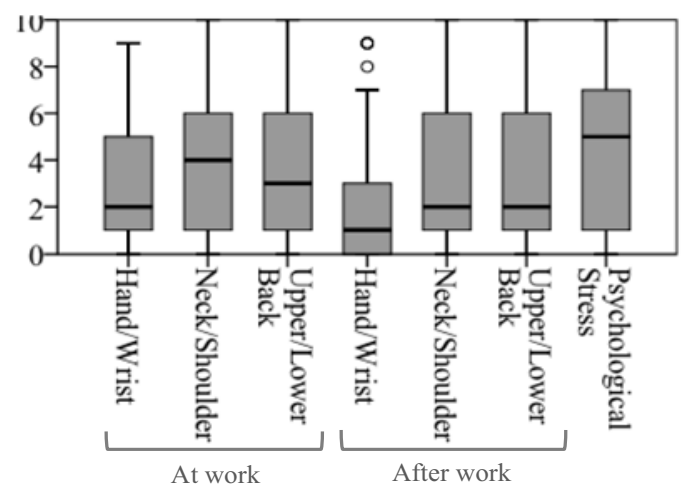

Fig 1. Mean pain/discomfort and psychological stress scores during and after work

\subsection{Associations between WMSD outcomes and demographic variables}

Non-parametric correlation analyses (Table 2) revealed that gender was significantly correlated with work stress $(\mathrm{r}=0.292, p=0.013)$ and existing health problems was significantly associated with increased pain/discomfort score during work for neck/shoulder $(\mathrm{r}=-0.216, p=0.038)$. A paired-t test revealed that females reported higher perceptions of work stress 
compared to males $(p=0.009)$. No other demographic variable was significantly associated with pain and stress scores.

\subsection{Associations between WMSD outcomes and JCQ categories}

In general, all categories of the JCQ (except decision authority) were significantly correlated to work stress score (Table 2). Increased psychological demands and physical exertions were associated with increased perception of work stress (psychological demand: $\mathrm{r}=0.368, p<0.0001$; physical exertion: $\mathrm{r}=0.284, p=0.005)$. Higher work stress was also associated with lower skill discretion $(r=-0.279$, $p=0.006)$ at work and limited supervisor support $(\mathrm{r}=-0.224, p=0.031)$.

Pain/discomfort scores (during and after work) of the three different body parts were significantly correlated to three JCQ categories, namely, physical exertion, supervisor support, and co-worker support (Table 2). Increased physical exertion, and limited support from co-workers and supervisor were associated with higher pain/discomfort scores during and after work for all body parts (hands/wrist, neck/shoulder, and upper/lower back).

\subsection{Predictors of WMSD outcomes}

In order to better explore the relationship between work and environment factors and WMSD outcomes, a step-wise multiple regression analysis was performed. Demographic variables (such as gender, age, daily work hours, number/duration of breaks, and existing health problems) and the six JCQ categories were used to predict pain/discomfort and work stress scores. Results are presented in Table 3. In general, the most important predictor of WMSD outcomes was co-worker support. Decision authority and psychosocial demands were also found to be important predictors of pain/discomfort scores during work and work stress. Demographic variables (gender, age, frequency of breaks, and existing health problems) were found to be significant predictors of pain/discomfort scores after work. Finally, almost all JCQ categories were found to be strong predictors of work stress. It is of interest to note that supervisor support was not found to be a significant predictor for most pan/discomfort scores and work stress.

Table 2

Correlations between demographic and JCQ categories and WMSD outcomes

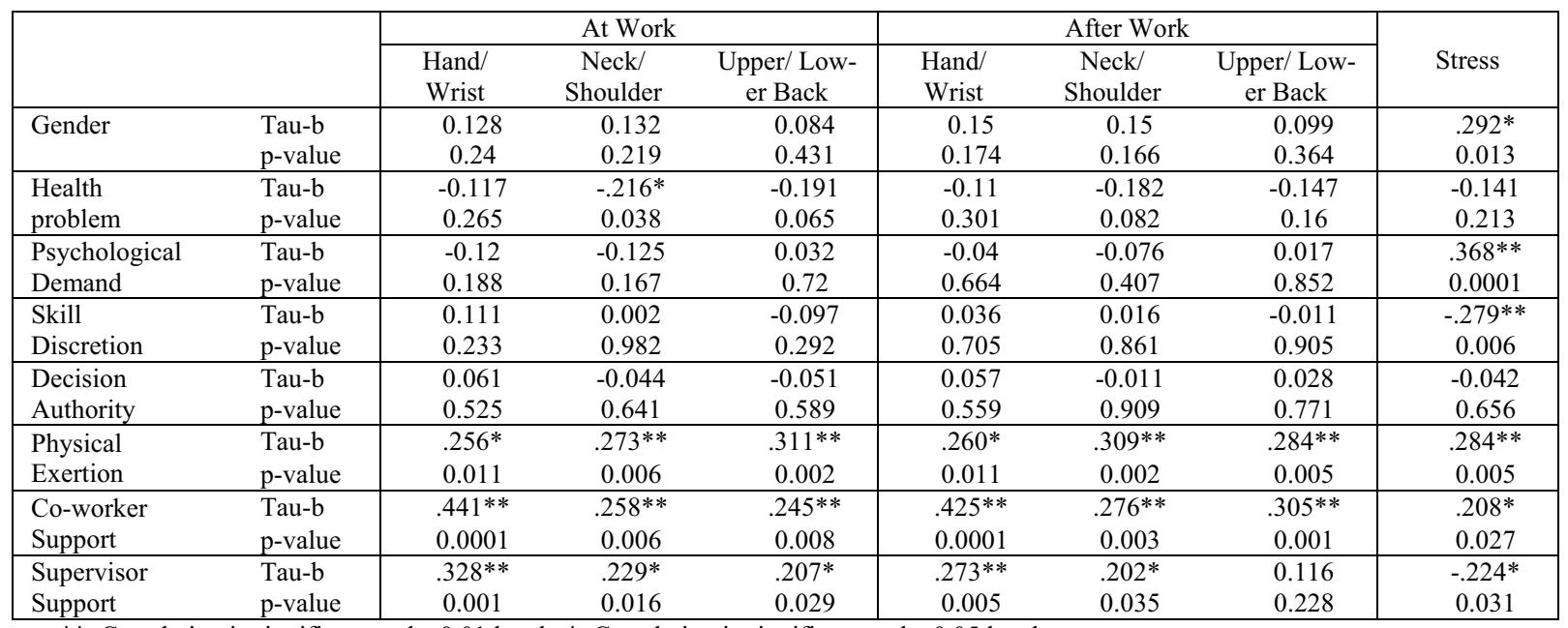

**. Correlation is significant at the 0.01 level, *. Correlation is significant at the 0.05 level 
Table 3

Multiple regression analyses

\begin{tabular}{|c|c|c|c|c|c|c|c|}
\hline & \multicolumn{3}{|c|}{ At Work (Beta-values) } & \multicolumn{3}{|c|}{ After Work (Beta-values) } & \multirow[b]{2}{*}{$\begin{array}{c}\text { Stress } \\
\text { (Beta- } \\
\text { values) }\end{array}$} \\
\hline & $\begin{array}{l}\text { Hand/ } \\
\text { Wrist }\end{array}$ & $\begin{array}{l}\text { Neck/ } \\
\text { Shoulder }\end{array}$ & $\begin{array}{l}\text { Upper/ Low- } \\
\text { er Back }\end{array}$ & $\begin{array}{r}\text { Hand/ } \\
\text { Wrist }\end{array}$ & $\begin{array}{l}\text { Neck/ } \\
\text { Shoulder }\end{array}$ & $\begin{array}{c}\text { Upper/ } \\
\text { Lower } \\
\text { Back }\end{array}$ & \\
\hline Age & & & & & 0.261 & & \\
\hline Number of breaks & & & & & 0.512 & & \\
\hline Duration of breaks & & & & & & & 1.507 \\
\hline $\begin{array}{l}\text { Psychological } \\
\text { demands }\end{array}$ & & & 0.113 & & & & 0.046 \\
\hline Skill Discretion & & & & & & & -0.162 \\
\hline Decision Authority & & -0.108 & -0.123 & & & & -0.033 \\
\hline Physical Exertion & & & & & & & 0.772 \\
\hline Co-worker Support & 0.301 & 0.344 & 0.364 & 0.256 & 0.216 & 0.467 & 0.214 \\
\hline
\end{tabular}

\section{Discussion}

The purpose of the study was to identify and assess the contributions of prevalent psychosocial factors on perceived WMSD outcomes among IT professionals from India. The findings suggest prevalence of existing pain (shoulder/neck and low back) in more than one-fourth of the respondents. Of all the scales of JCQ, co-worker support was the strongest (and most consistent) predictor of pain/discomfort scores and psychological stress. Psychological stress was strongly correlated to all JCQ scales (except decision authority). The results suggest a greater association between psychosocial risk factors and WMSD outcomes than physical demands. Thus, it is important to include multiple dimensions of demand (i.e., physical, organizational, psychological) when assessing worker health and well being in different occupations.

To date, this is one of the few studies that employed the JCQ to assess psychosocial strains in an Indian population. Mohan et al. [19] reported a greater influence of hazardous working conditions and limited decision making authority on work strain in Indian foundry shop floor workers. The difference in the work demands (both physical and psychosocial) between the occupational settings in their study to the current study is argued to drive differences between the outcomes. The information technology work cul- ture is a representation of modern office, with a greater influence of the American work culture characterizing the IT culture. It is not surprising, as the IT companies prevalent in India are international branches of the American counterparts.

One of the major predictors of WMSD outcomes obtained in this study was limited co-worker support. Work support has been shown to moderate the effects of stress [10] and result in enhanced performance at work [20]. A recent study by Surana et al. [25] demonstrated an inverse relationship between social support and job burnout among call center workers in India. Unlike this study, the current study did not observe a strong relationship between supervisor support and psychological stress. It may be possible that the respondents were reluctant to answer the question items related to supervisor support.

Lack of decision authority and skill discretion and increased psychological demands were found to influence pain/discomfort and psychological stress scores. These findings are consistent with previous studies where workers were constrained at their jobs with respect to making decisions or learning new things [19]. Furthermore, increased psychological demands can result in gradual strain leading to musculoskeletal symptoms and pain [18]. Psychosocial risk factors can influence musculoskeletal pain by: 1) varying biomechanical demands associated with the job, 2) increasing awareness of pain symptoms, or 3) adversely affecting physiological attempts at recov- 
ery. These mechanisms may be potentially explained by physiological pathways such as increased muscle tension [17], altered joint kinematics [8], interference with blood flow and energy metabolism [29], and changes in blood catecholamine levels [16].

Of most of the demographic variables, only gender and existing health problems were correlated to pain/discomfort and psychological stress scores. However, it is of interest to note that about two-thirds of the respondents had never had any ergonomics awareness training. This can be explained, in parts, to the lack of existing work-related health standards in India (specifically for modern office). Additionally, respondents indicated that they worked about 9 hours daily, with a maximum of 30 minutes breaks in between. Moreover, the frequency of these breaks ranged from 1-3 breaks daily. Existing research findings suggest that frequent small breaks are more beneficial in relieving localized muscular fatigue when compared to infrequent longer breaks [2]. Interventions such as these should thus be incorporated to relieve any pain/discomfort during work, and greater emphasis should be placed on conducting organization-wide ergonomics training.

One of the limitations of this study is the low sample size. However, this was a first attempt at employing the JCQ to the IT industry among Indian professionals. Results from this pilot is expected to fuel a larger study design, which will focus on deriving subjective and physiological correlates of work stress among IT professionals in India. In conclusion, findings from this study highlight the influence of certain psychosocial traits of the Indian IT workplace on WMSD outcomes. Intervention strategies can be designed and implemented to address these factors that may help lower the risk of work-related musculoskeletal pain.

\section{References}

[1] Ariëns GAM, van Mechelen W, Bongers PM, Bouter LM, van der Wal G. Psychosocial risk factors for neck pain: A systematic review. American Journal of Industrial Medicine. 2001;39:180-93.

[2] Balci R, Aghazadeh F. Effects of exercise breaks on performance, muscular load, and perceived discomfort in data entry and cognitive tasks. Computers \& Industrial Engineering. 2004;46(3):399-411.

[3] Bhanderi D, Choudhary S, Parmar L, Doshi V. Influence of psychosocial workplace factors on occurrence of musculoskeletal discomfort in computer operators. Indian J Community Med. 2007;32(3):225-6.

[4] Bhanderi D, Choudhary S, Parmar L, Doshi V. Influence of psychosocial workplace factors on occurrence of musculoskeletal discomfort in computer operators. Indian J Community Med. 2007 July 1, 2007;32(3):225-6.

[5] Borg G. Psychophysical scaling with applications in physical work and the perception of exertion. Scandinavian Journal of Work, Environment \& Health. 1990;16:55.

[6] Buckle PW. Upper limb disorders and work: the importance of physical and psychosocial factors. J Psychosom Res. 1997;43(1):17-25.

[7] Chyuan J-YA, Du C-L, Yeh W-Y, Li C-Y. Musculoskeletal disorders in hotel restaurant workers. Occupational Medicine. 2004 January 1, 2004;54(1):55-7.

[8] Faucett J, Rempel D. VDT-related musculoskeletal symptoms: interactions between work posture and psychosocial work factors. American Journal of Industrial Medicine. 1994;26(5):597-612.

[9] Feuerstein M, Nicholas R. Development of a short form of the workstyle measure. Occup Med (Lond). 2006;56(2):94-9.

[10] Gray Stanley J, Muramatsu N, Heller T, Hughes S, Johnson T, Ramirez Valles J. Work stress and depression among direct support professionals: the role of work support and locus of control. Journal of Intellectual Disability Research. 2010;54(8):749-61.

[11] Hart SG, Staveland LE. Development of NASA-TLX (Task Load Index): Results of empirical and theoretical research. In: Hancock PA, Meshkati N, editors. Human Mental Workload. Amsterdam: North-Holland; 1988. p. 139-83.

[12] Huang GD, Feuerstein M, Sauter SL. Occupational stress and work-related upper extremity disorders: Concepts and models*. Am J Ind Med. 2002;41(5):298314.

[13] Karasek RA. Job Content Questionnaire and User's Guide. Lowell, MA: University of Massachusetts Lowell, Department of Work Environment1985.

[14] Karasek RA, Brisson C, Kawakami N, Houtman I, Bongers PM. The Job Content Questionnaire (JCQ): An instrument for internationally comparative assessments of psychosocial job characteristics. Journal of Occupational Health Psychology. 1998;3:322-55.

[15] Landsbergis PA, Theorell T, Schwartz JE, Greiner BA, Krause N. Measurement of psychosocial workplace exposure variables. Occupational Medicine. 2000;15:163-88.

[16] Lundberg U. Psychophysiology of work: Stress, gender, endocrine response, and work-related upper extremities disorders. American Journal of Industrial Medicine. 2002;41:383-92.

[17] Lundberg U, Forsman M, Zachau G, Ekloèf $M$, Palmerud G, Melin B, et al. Effects of experimentally induced mental and physical stress on motor unit recruitment in the trapezius muscle. Work \& Stress. 2002;16(2):166-78.

[18] Melin B, Lundberg U. A biopsychosocial approach to work-stress and musculoskeletal disorders. Journal of Psychophysiology. 1997;11:238-47.

[19] Mohan G, Elangovan S, Prasad P, Krishnaaan P, Mokkapati A. Prevalence of job strain among Indianfoundry shop floor workers. Work. 2008;30:3537.

[20] Nagami M, Tsutsumi A, Tsuchiya M, MORIMOTO K. Job Control and Co-worker Support Improve Employee 
Job Performance. Industrial health 2010(0):1006240012.

[21] NRC. Musculoskeletal disorders and the workplace: Low back and upper extremities. 2001.

[22] Reid GB, Nygren TE. The subjective workload assessment technique: A scaling procedure for measuring mental workload. In: Hancock PA, Meshkati $\mathrm{N}$, editors. Human Mental Workload. Amsterdam: North Holland; 1988. p. 185-218.

[23] Sharan D, Parijat P, Sashidharan P, Ranganathan R, Mohandoss M, Jose J. Workstyle Risk Factors for Work Related Musculoskeletal Symptoms Among Computer Professionals in India. JOR. 2011.

[24] Sharma A, Khera S, Khandekar J. Computer related health problems among information technology professionals in Delhi. Indian $\mathrm{J}$ Community Med. 2006;31(1):36-8

[25] Surana S, Singh AK, Saxena S. The management of job burnout among call centre customer service agents in India: the role of social support. International Journal of Management Development. 2011;1(1):79-97.

[26] Talwar R, Kapoor R, Puri K, Bansal K, Singh S. A study of visual and musculoskeletal health disorders among computer professionals in NCR Delhi. Indian Journal of Community Medicine. 2009;34(4):326-8.
[27] Toomingas A, Theorell T, Michelsen H, Nordermar R. Associations between self-rated psychosocial work conditions and musculoskeletal symptoms and signs. Scand J Work Environ Health. 1997;23(2):130-9.

[28] Wahlström J, Hagberg M, Toomingas A, Wigaeus Tornqvist E. Perceived muscular tension, job strain, physical exposure, and associations with neck pain among VDU users; A prospective cohort study. Occupational and Environmental Medicine. 2004;61:523-8.

[29] Warren N. Work stress and musculoskeletal disorder etiology: The relative roles of psychosocial and physical risk factors. Work: A Journal of Prevention, Assessment and Rehabilitation. 2001;17(3):221-34.

[30] Warren N. Work stress and musculoskeletal disorder etiology: The relative roles of psychosocial and physical risk factors. WORK. 2001;17(3):221-34.

[31] WHO. Identification and control of work-related diseases : Report of a WHO expert committee. Albany, NY: Geneva: World Health Organization; 1985. 